\title{
DESIGN AND DEVELOPMENT OF A RAMAN LIDAR FOR CHERENKOV GAMMA ARRAY EXPERIMENTS
}

\author{
George Vasileiadis ${ }^{{ }^{*}}$, Patrick Brun ${ }^{1}$, Omar Gabella ${ }^{1}$, Stephane Rivoire ${ }^{1}$, George Avdikos ${ }^{2}$, \\ Alexandros Louridas ${ }^{2}$, George Georgoussis ${ }^{2}$ and Alexandros Papayannis ${ }^{3}$ \\ ${ }^{1}$ University of Montpellier 2, LUPM, Montpellier, FR34095, France \\ ${ }^{2}$ Raymetrics S.A., Metamorfossis, GR14452, Greece \\ ${ }^{3}$ National Technical University of Athens, Physics Department, Zografos, GR15780, Greece \\ *Email: george.vasileiadis@lupm.in2p3.fr
}

\begin{abstract}
Future Cherenkov Gamma Ray (CGR) experiments will reach a sensitivity and energy resolution never obtained until now by any other high energy gamma-ray experiment. It is well known that atmospheric conditions contribute particularly in this aspect. Raman lidars can help reduce the systematic uncertainties of the molecular and aerosol components of the atmosphere so these performances can be reached. The motivation for Raman lidars and the design and development of the Montpellier Raman lidar system is described. It provides both multiple elastic and Raman readout channels and custommade optics design. Preliminary lidar tests and signals show the actual performance of the lidar in consistency with the desired goals.
\end{abstract}

\section{INTRODUCTION}

Future Cherenkov Gamma Ray (CGR) experiments will reach a sensitivity and energy resolution never obtained until now by any other high energy gamma-ray experiment [1]. It is well known that atmospheric conditions contribute particularly in this aspect.

Current Imaging Atmospheric Cherenkov Telescope (IACT) experiments and the Pierre Auger Observatory have pioneered several ways to introduce atmospheric calibration devices, among them the use of Lidars [2,3]. Their experience has led to a coherent atmospheric calibration strategy for the future CGR experiments. One of its key components consists of the assessment of the atmospheric extinction profile throughout the entire path that Cherenkov photons may take before getting imaged, at two wavelengths within the sensitive regime of the light sensors used in CGR telescope cameras.

To achieve this, one or several Raman lidars, operating at various wavelengths will provide extinction profiles, to continuously assess the atmospheric extinction across the observed science targets. Such an approach is sufficiently precise when either a single or no cloud layer are present in the observed field-of-view, a situation fulfilled practically all the time when the CGR experiments will be operative.

To precisely characterize extinction profiles up to $25 \mathrm{~km}$ distance, the Raman lidars should use powerful lasers and large mirrors. These choices guarantees the highest precision at the expense of strong interference with the light sensors making it impossible to simultaneously operate both. Since the telescopes need to re-point frequently, the Raman lidars must therefore be able to measure the extinction profiles within short time scales (a few minutes).

The Laboratoire Univers et Particules de Montpellier (LUPM) is using a recycled $1.8 \mathrm{~m}$ mirror from the CLUE (Cherenkov Light Ultraviolet Experiment) experiment [4], to achieve the required sensitivity to backscattered light (Figure 1). A specific spectrometer (wavelength separation unit) has been designed and manufactured for the detection of signals at 355 , 387, 532 and 607nm. Zemax [5] and Solidworks [6] simulations have been applied, leading to a compact and robust spectrometer. Preliminary lidar tests and signals show the actual performance of the lidar in consistency with the desired goals.

\section{METHODOLOGY}

\subsection{The LUPM lidar system}

The LUPM Raman lidar is optically and mechanically based on a telescope and mechanical assembly from the CLUE former experiment. Due to the small cross section of the Raman lines, about 2 to 3 orders of magnitude smaller than the elastic cross section, a large reflecting area is needed to 
collect a sufficient amount of light. For this reason, the telescope reflector is $1.8 \mathrm{~m}$ in diameter and coated to optimize UV reflection. It is installed on an altazimuth mount. Measurements of the point spread function and reflectivity show that the mirror has maintained a good optical quality, although the reflectivity has decreased to $64 \%$ at $350 \mathrm{~nm}[7]$.

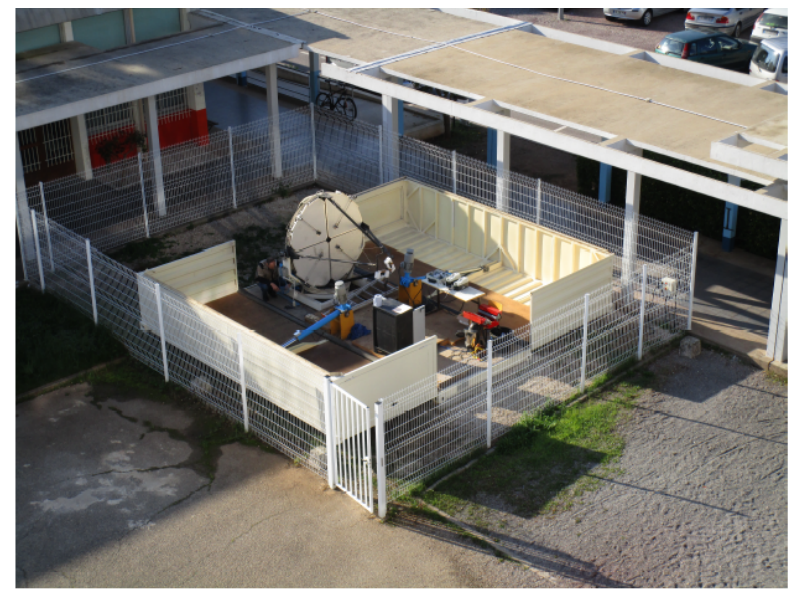

Figure 1. The refurbished CLUE container and UV coated mirror used for the Raman LIDAR.

Pointing the telescope to a specific direction is achieved by a pair of stepper motors with a precision of 0.02 degrees. The motors are piloted by a dedicated automation system built around a Panasonic PF7 PLC. A Quantel CFR 400 Nd:YAG laser is mounted at a fix point on the telescope's structure. It is a military-grade III $9 \mathrm{~ns}$ pulsed laser, with a repetition rate of $20 \mathrm{~Hz}$, providing three different wavelengths at $1064 \mathrm{~nm}, 532 \mathrm{~nm}$ and 355 $\mathrm{nm}$ in a coaxial configuration, in order to achieve a good overlap between the laser beam light cone and the telescope field of view.

A good overlapping factor is needed to measure the optical depth starting from a few tens of meters height. This is important since measuring the extinction of the atmosphere as low as possible permits a better estimation of the photon absorption.

The laser beam exits the telescope using a double UV-coated mirror guiding system. The laser head is firmly mounted on the telescope dish. A single 1-inch fixed mirror will guide the laser beam to the center of the dish at the level of the focal point. To achieve optimal performance, a precise alignment system is needed to assure that at any moment the optical axis of the telescope and the laser beam are co-linear. We have opted for a computerized industrial system by ThorLabs, with a step-size precision of less than $1 \mathrm{~mm}$.

A support for the light guide has also been installed in the structure of the telescope. The pieces fix it to the structure in such a way that movements are restricted and a minimum bending radius for the liquid fiber light guide is assured.

\subsection{The spectrometer system}

The light output from the focal point of the telescope is fed to the entrance of the Raman polychromator via a Lumatec 300 series liquid fiber of $8 \mathrm{~mm}$ diameter with a numerical aperture of 0.59 . We use this kind of fiber to avoid stress effects due to bending during the pointing phase of the telescope, while keeping an excellent UV transmission (better than 70\%). Other solutions based on custom rigid optical components were studied but did not have good enough transmission. Figure 2 presents the polychromator built by the Raymetrics company.

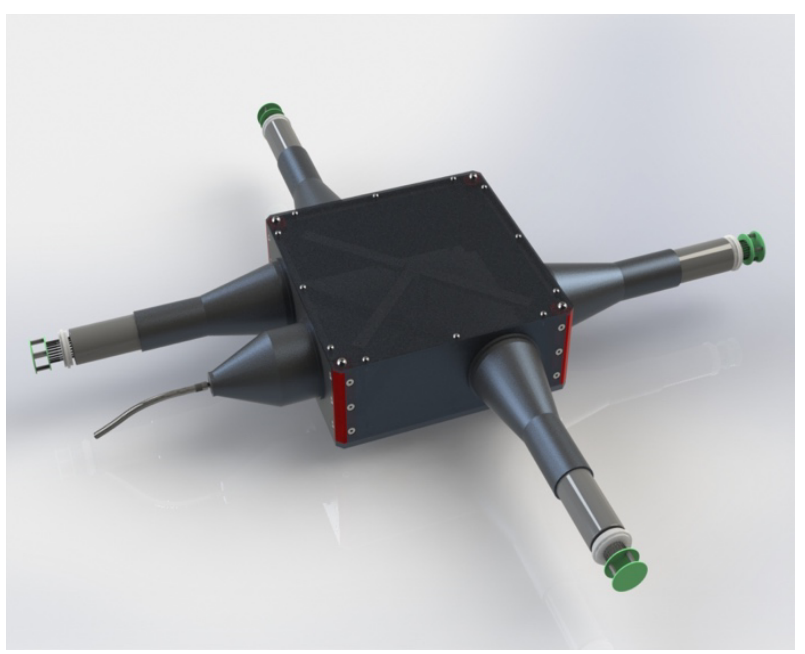

Figure 2. Image of the Raymetrics Raman spectrometer. A four channel Elastic/Raman design with a liquid light guide input option.

The polychromator is designed to be lightweight, mechanically modular, and optically efficient. Dichroic mirrors are used to separate the different Raman and elastic lines, while a dedicated two-lens eye piece is used in front of every PMT to focus uniformly the incident light. Custom 2-inch optics 
are used throughout the spectrometer to match efficiently the PMT entrance window size.

\section{RESULTS}

\subsection{Optical design simulations}

In order to efficiently simulate the image formation from near and far end achieving 80\% coverage at the PMT photocathode level (considering the specifications of the big telescope), it was necessary to use 2-inch optical components. A set of 2-inch collimating lenses at the output of the fiber was used for collimating the beam and inserting this into the spectrometer. Then, specific custom dichroic beam splitters (CVI) were used for the separation of the wavelengths into different directions. Also 2-inch interference filters with FWHM equal to $10 \mathrm{~nm}$ were used in front of every 2-inch Hamamatsu R329P Photomultiplier Tubes (PMT). Simulations with Zemax software shown that there is a very homogeneous distribution of the image for both near and far field into the PMT cathode, almost eliminating all redundant effects caused by PMT inhomogeneities (Figure 3).

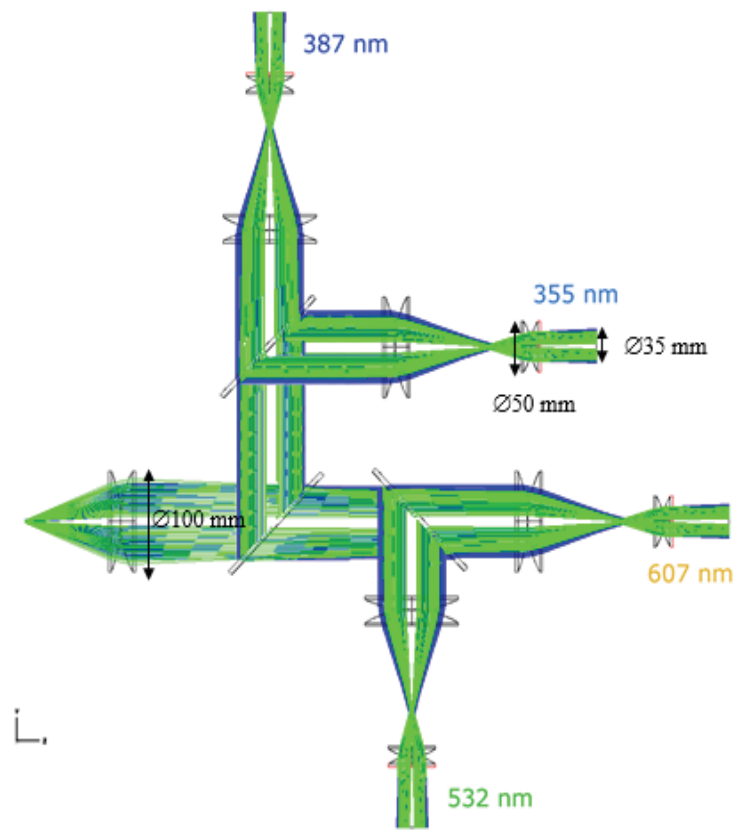

Figure 3. Zemax simulations of the spectrometer.

\subsection{Expected performance of the Raman Lidar}

Studies of the PSF and reflectivity of the telescope mirror, show that it has maintained a good optical quality, keeping basically the same PSF as when it was produced $(6.5 \mathrm{~mm})$, although the reflectivity has decreased to $64 \%$ at $350 \mathrm{~nm}$. The mirror spot size has been characterized through its PSF by different methods. Different energy containments at different radii are calculated. The results obtained show that $80 \%$ of the light is contained in an area of $6 \mathrm{~mm}$ diameter while the $90 \%$ falls in a circle with a diameter of about $6.4 \mathrm{~mm}$ (Figure 4 ).

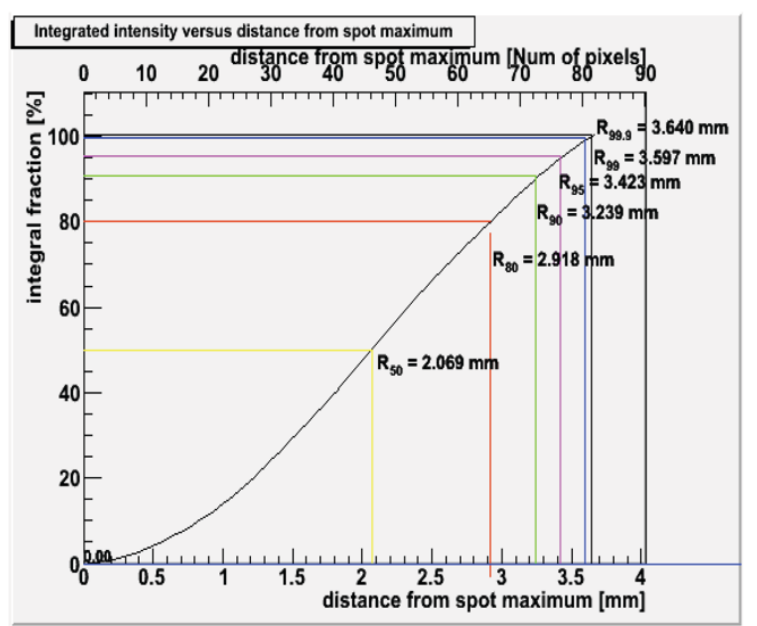

Figure 4: Measurement of the primary mirror PSF. All light is enclosed in the liquid light guide perfectly. In this case, D90 around $6.4 \mathrm{~mm}$.

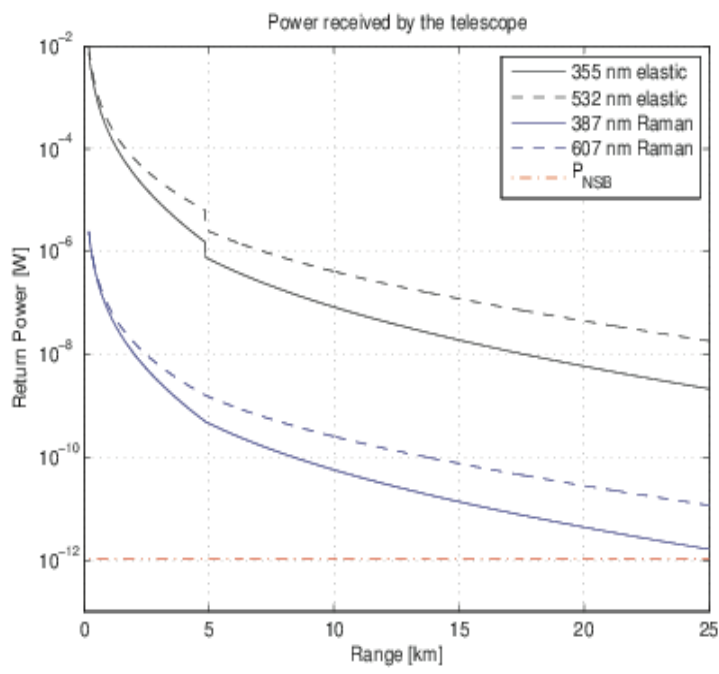

Figure 5: Estimated return power from the link-budget simulation of the Raman LIDAR. The horizontal solid red line is the background power calculated for typical NSB levels.

Finally, dedicated link-budget simulations [8] have revealed that this system can characterize the atmosphere up to $25 \mathrm{~km}$ with the elastic channel, 
and up to $18 \mathrm{~km}$ with the Raman channels in less than a few minutes (see Fig. 5).

Preliminary return signal profiles are also shown at Figure 6. Only results obtained using the $532 \mathrm{~nm}$ line are presented here. Even if we are still in the testing phase these first results are very much promising for the future. A loss of signal is observed at lower altitudes due to misalignment issues, under investigation, while the performance at high altitudes is as expected.

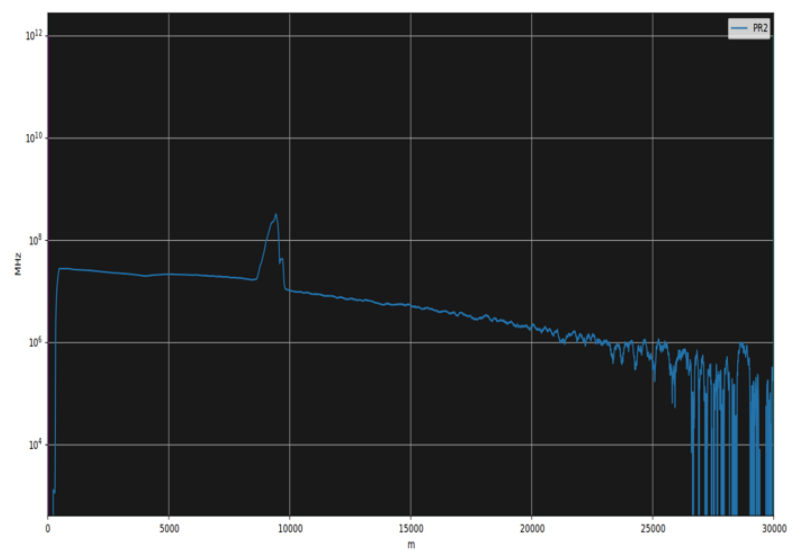

Figure 6: Preliminary results obtained using the $532 \mathrm{~nm}$ laser line. The log of the return signal, range corrected, is plotted against altitude.

\section{Conclusions}

For future CGR experiments a proper characterization of the atmosphere has to be done, in order to estimate the extinction that the UV photons undergo from the top of the atmosphere to the ground and correctly reconstruct the energy of the primary gamma ray. A suitable device to achieve this goal is a Raman LIDAR, an instrument that can provide a height resolved characterization of the atmosphere. The Raman LIDAR would allow to correct systematic biases on the energy scale and flux, reducing the systematic uncertainties from a current $20 \%$ down to better than $5 \%$. This will help not only to reach the desired energy resolution but also to increase the duty cycle.

Preliminary results both on simulations and actual expected performance are promising. The optical system is currently under assembly and first test bench confirms system requirements. As soon as the behaviour of the complete chain is completely understood the LIDAR will be operational as a full Raman one.

\section{References}

1. Actis, M. et al. Design concepts for the Cherenkov Telescope Array CTA: an advanced facility for ground-based highenergy gamma-ray astronomy, Exp. Astron. 32193 (2011)

2. Will M. Atmospheric Monitoring at the Site of the MAGIC Telescopes, Proc. AtmoHEAD 2016, EPJ 14401002 (2017)

3. G.Vasileiadis et al. An elastic lidar system for the H.E.S.S. NIM A819(2016)60-66

4. Alexandreas D. et al. Status report of CLUE NIM A 360385 (1995)

5. www.zemax.com

6. www.solidworks.com

7. A.Oramas et al. ICRC 2013 (1517).

8. M. Eizmendi, Master Thesis (2011) 